\title{
BLIND-SPOT DETECTION WITH AUTOMATIC STEERING
}

\author{
A.Kula Sekhar ${ }^{1}$, Nishad Nazar ${ }^{2}$ \\ ${ }^{1}$ M.Tech, Sensor System Technology dept, VIT University, Vellore, Tamil Nadu, India \\ ${ }^{2}$ M.Tech, Sensor System Technology dept, VIT University, Vellore, Tamil Nadu, India
}

\begin{abstract}
Present days, the automotive industry is mainly focusing on the road safety measures. The automobiles have been constantly updating with new sensing technologies to detect blind spots, which can prevent road accidents. Almost 1.32 million people die in road accidents each year. Blind spot of an automobile is the region of the vehicle which cannot be observed properly while looking either through side or rear mirror view. To meet the above requirements, this paper describes detecting blind spot by using ultrasonic sensor and controlling the direction of car by automatic steering. The technology embedded in the system is capable of automatically steer the vehicle away from an obstacle if the system determines that a collision is impending or if the vehicle is in the vicinity of our car.
\end{abstract}

Keywords -- Arduino Mega 2560, Ultrasonic Sensor, L293 Motor IC $* * *$

\section{INTRODUCTION}

In the present days the toll of road accidents has been in the increasing graph and many accidents are caused due to negligence of the driver. Most of the accidents also happen in the highway driving, where driver have negligence during lane departure/switching of vehicles. Thus, detecting the obstacle near the vicinity of our vehicle during lane switching/departure or during highway driving is vital and many research and development is still under process to make the vehicle safer to drive and control it automatically during these situations. The advanced automotive technology has been developing better systems to enhance the driver safety. The automotive technology is always on the thought of enhancing and bettering the safety of the vehicle than their predecessors. Automobile manufactures have proposed any safety schemes till date, like, collisionforewarning technology, lane departure warning system (LDWS), Blind spot information systems, vehicle back assist systems.

This paper describes about the driver negligence at the blind spot, and detecting the obstacle in order to automatically steer the car by providing partial braking to the individual wheels. Blind spot is the area where the driver cannot observe properly during driving due to many factors like head rest, pillar obstacle, passenger height etc., and many accidents occur on the blind spot and hence this area needs to be monitored and obstacle needs to be detected. Automotive technology has developed Blind Spot Information System (BLIS) and it was first developed by the giant car manufacturer Volvo in the year 2007. This system detects any obstacle like pedestrian crossing suddenly, cars overtaking etc. and the driver is alerted. The system uses two door mounted lenses to check the blind spot for an impeding collision on the vicinity of the vehicle. The overtaking of vehicles is a big factor were the accidents happen on the blind spot area. The mirrors become helpless during this situation and there could be a technology to avoid the collision on the blind spot during overtaking, lane switching etc. The system uses the ultrasonic sensors around the vehicle to detect the incoming obstacle in the specified range and sensor feedback is given to the Arduino microcontroller as to control the motors connected to wheels to reduce the speed when an obstacle is detected, the decreasing of speed or partial braking as in.

The different sections in the paper will explain the different aspects of the system. Section II explains about the hardware description, Blind Spot detection is briefed in section III, section IV describes the working of the system,

\section{HARDWARE SETUP}

The system is based on Arduino microcontroller. The system consist ultrasonic sensor, motor IC, DC motor. The obstacle when detected in the blind spot, the data is acquired by ultrasonic sensor HC-SR04 with a specified range and then it is fed to the input for Arduino mega 2560. The ultrasonic sensor measures the time difference of the obstacle in the vicinity of the vehicle and then calculates the distance with time. Then the data is manipulated to control the motors and to reduce the speed of motor by specified amount.

\section{BLIND SPOT DETECTION}

A Collision Avoidance system is that a safety to the automobile system to reduce the immense damage and to reduce the effect of the accident. It is also known as Precrash system; the system rather uses radar or Ultrasonic sensors and also nowadays the LIDAR technology is also being used as to implement the system. All the safety schemes can be put under the collision avoidance system. And this system can be used in the Blind spot techniques. When the vehicle gets into the blind spot region, then the sensor detects the obstacle and the data is used to alert or manipulate the vehicle for the safety measure. And the improvised version of Blind Spot monitoring is been used in 
Blind Spot Information System, developed and implemented in Volvo S80 Sedan class in 2007. The car being fixed with two lenses which is mounted to check the Blind Spot region for an impending collision. Once obstacle detected it is send to the processing unit and then alerted the system.

The system is imposed with Arduino Mega 2560 microcontroller board, once the obstacle is detected, the data acquired by the sensor is used to process the distance and then it is used to control the four Dc motors connected to the individual four wheels of the vehicle. The motor is driven by the L293D motor IC.

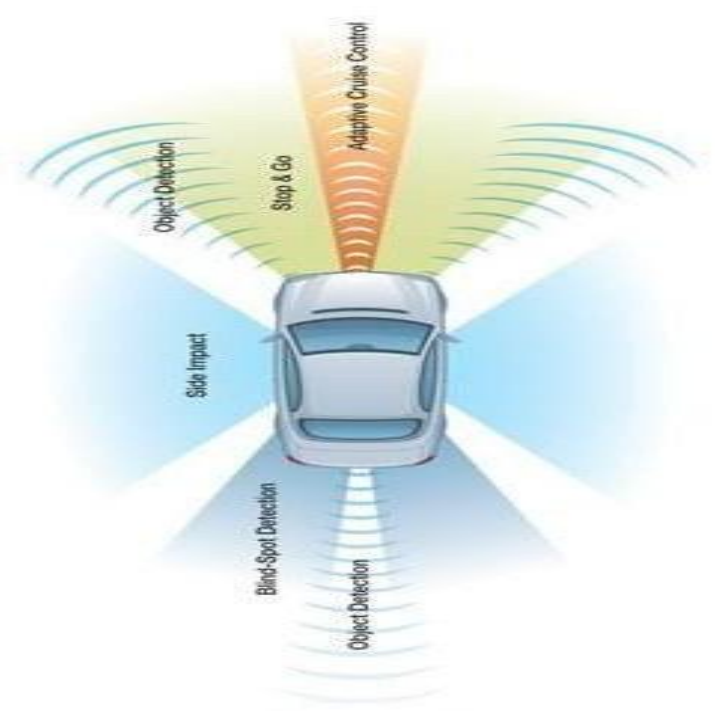

\section{WORKING}

Blind spot is an region surrounding the vehicle which cannot be observed properly by the driver if there is an obstacle like head rest, passenger height, window pillar etc. The vehicle is been fitted with four sensors to check the front, rear, and the two side $\mathrm{s}$ of the automobile. When an obstacle is detected on the blind spot area, the distance of the obstacle is calculated and fed to the Arduino Mega to process the necessary action. The ultrasonic sensor HC-SR04 provides $2 \mathrm{~cm}-400 \mathrm{~cm}$ measurement range, the accuracy of ranging can reach to $3 \mathrm{~mm}$.

The principle of the sensor is

[1] By the Use of the IO trigger pin for a 10us high level signal.

[2] Then the Module automatically transmits eight $40 \mathrm{kHz}$ and when obstacle is detected, a pulse wave is transmitted back.

[3] If the signal return is received at the echo pin, through high level, the time of high output level is the duration from the ultrasonic to return.

The formula being used is

Distance $(\mathrm{d})=($ high level time $(\mathrm{t}) \times$ velocity of sound $(\mathrm{v})$ $(340 \mathrm{M} / \mathrm{S}) / 2$; or $\mathrm{uS} / 58=$ centimeters or $\mathrm{uS} / 148=$ inch.
After calculating the distance of the obstacle, the data is provided to the Arduino Mega 2560 processing unit. The arduino is programmed such that, if an obstacle is detected near to the distance specified then the motors are controlled to reduce the speed of the motor to control the wheel speed or the speed of the vehicle. Initially, the wheels are running on a constant speed. We are connecting four Ultrasonic sensors on the four locations; front, rear, and two sides of the vehicle. So we have different cases to manipulate the vehicle.

Case 1: If any obstacle is detected by sensor on either of the side, then the opposite set of wheels will have reduced speed provided by the controller.

Case 2: If any object obstacle is detected by the sensor on the rear, then the speed of the rear wheels are increased.

Case 3: If any obstacle in the blind spot area is detected by the sensor on the front, then the speed of all wheels are decreased.

All the motors are controlled by the L293D motor IC. Each IC will control each motor corresponding to the wheel connected. The speed being decreased can be focussed as partial braking; the system detects and reduces speed as the result as partial braking is focused.

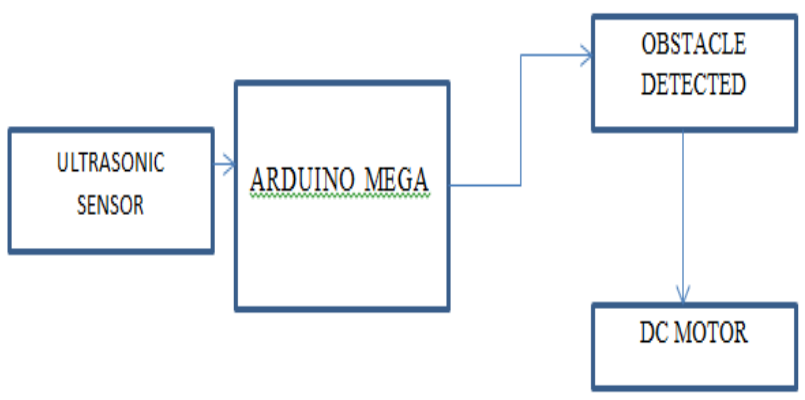

\section{CONCLUSION}

Thus, by the following system developed, the vehicle detects the blind spot area and then controls the speed of the individual motors connected to the axial of the wheel. As per the National Highway Authority of India (NHAI), road accidents occur mostly due to overtaking in the highway due to the negligence of the driver. And therefore, with this kind of system developed, the accidents caused due to driver negligence can be brought down by an extent.

\section{REFERENCES}

[1]. A. Techmer, "Real-time Motion Analysis for Monitoring the Rear and Lateral Road", Proceedings of 2004 IEEE Intelligent Vehicles Symposium, Vol. 11, pp. (11)704- 709 .

[2]. R. Sosa and G. Velazquez, "Obstacles detection and collision avoidance system developed with virtual models," in Proc. IEEE Int. Conf. on Vehicular Electronics and Safety, Beijing, China, Dec. 13-15, 2007, pp. 1-8.

[3]. Chen, C., Chen, Y.: Real time approaching vehicle detection in blindspot area. In: Intelligent Transportation Systems, 2009. ITSC '09. 12th International IEEE Conference on. pp. $1-6$ (2009) 